Take the online multiple choice questions associated with this article (see page 1104)
Correspondence to: Dr Jasbir Sra, University of Wisconsin Medical School-Milwaukee Clinical Campus, Milwaukee, Wisconsin, USA; bdanek@ hrtcare.com mage registration is the process of aligning images. ${ }^{12}$ During interventional procedures to deliver electrical therapy, the catheters or leads are usually manipulated under the guidance of a fluoroscopy system. Typically, as in the case of ablation of atrial fibrillation (AF), the most complex of the cardiac arrhythmias, mapping and ablation catheters must be manipulated in and around the pulmonary veins (PVs) and the left atrium (LA). ${ }^{3-5}$ These anatomical structures are not visualised by $x$ ray fluoroscopy as they do not present contrast against the surrounding anatomical structures. Currently available cardiac mapping systems can record catheter location, cardiac electrical activation information, and lesion location. ${ }^{6-9}$ However, these systems cannot reproduce complex cardiac chamber anatomy such as the LA-PV junction.

Segmentation of various body organs can be performed by such imaging techniques as computed tomography (CT) and magnetic resonance imaging. ${ }^{10-12}$ However, these imaging systems are not capable of tracking a catheter in real time or recording electrical activation. Combining the explicit three dimensional (3D) anatomy of a cardiac chamber such as the LA with the electrical and navigational information obtained with an interventional system, through a process known as registration, should increase the efficacy of ablation procedures.

Although various other modalities have been used in an attempt to delineate cardiac structures, 3D imaging of the LA using CT will be discussed in detail in this review. Currently, there is little application of 3D image registration in the treatment of cardiac arrhythmias. This review will describe the methods available for cardiac image registration in an attempt to give the reader a grasp of the main concept of registration without getting into great detail on the mathematical constructs underlying this evolving new technology.

\section{HISTORICAL PERSPECTIVE}

In 1895, in Birmingham, England, soon after the discovery of the $x$ ray, a casualty officer successfully guided his scalpel to a broken needle in the hand by aligning the $x$ ray plate with the hand of the patient. ${ }^{13}$ Over the last few decades, a stereotaxic frame was combined with CT and used in neurosurgery to locate targeted structures. ${ }^{14}$ However, the frame had to be attached before surgery and left on throughout the procedure. These problems were overcome with the development of more advanced, frameless surgery. ${ }^{15}$ Detailed atlases and computer models are now being used to perform registration for the treatment of some neurological problems. Despite rapid advances in computer technology, and the use of advanced algorithms in neurosurgery, only recently has interest shifted to the potential of 3D cardiac imaging and registration. Because of significant cardiac and respiratory motion, the registration process for cardiac studies is much more complex and challenging. However, the unique features of cardiac anatomy, the use of catheters positioned in various cardiac structures during interventional procedures, intracardiac recordings from these catheters, and the ability to inject and visualise contrast in structures such as the pulmonary veins provide a viable platform for registering these anatomical structures.

\section{SEGMENTATION AND RECONSTRUCTON OF LEFT ATRIAL IMAGES}

Detailed 3D reconstruction of an anatomical model is a prerequisite to any successful registration. Typically, medical images must be in a digital format to be registered. Most medical images are made up of pixels, which are small two dimensional arrays of square or rectangular elements, each having an associated image intensity value. These arrays include the coordinate system of the image. If a CT slice is made up of $512 \times 512$ pixels, each pixel will correspond to an element of the cut through the patient measuring about $0.5 \times 0.5 \mathrm{~mm}^{2}$. These individual images, called axial slices, are then stacked together to form a three dimensional volume. Each individual element in the 3D array, now called a voxel, corresponds to a small volume of tissue. Thus, if the slice spacing is $1.5 \mathrm{~mm}$, the voxel size is $0.5 \times 0.5 \times 1.5 \mathrm{~mm} .^{3}$ Some images can now be acquired directly as a $3 \mathrm{D}$ volume. In the case of magnetic resonance brain images, for example, voxel size may be $0.9 \times 0.9 \times 5 \mathrm{~mm}$ with $256 \times 256$ pixels in a slice. Magnetic resonance images may also be acquired as cubic voxels of $1.0 \times 1.0 \times 1.0 \mathrm{~mm}$. 

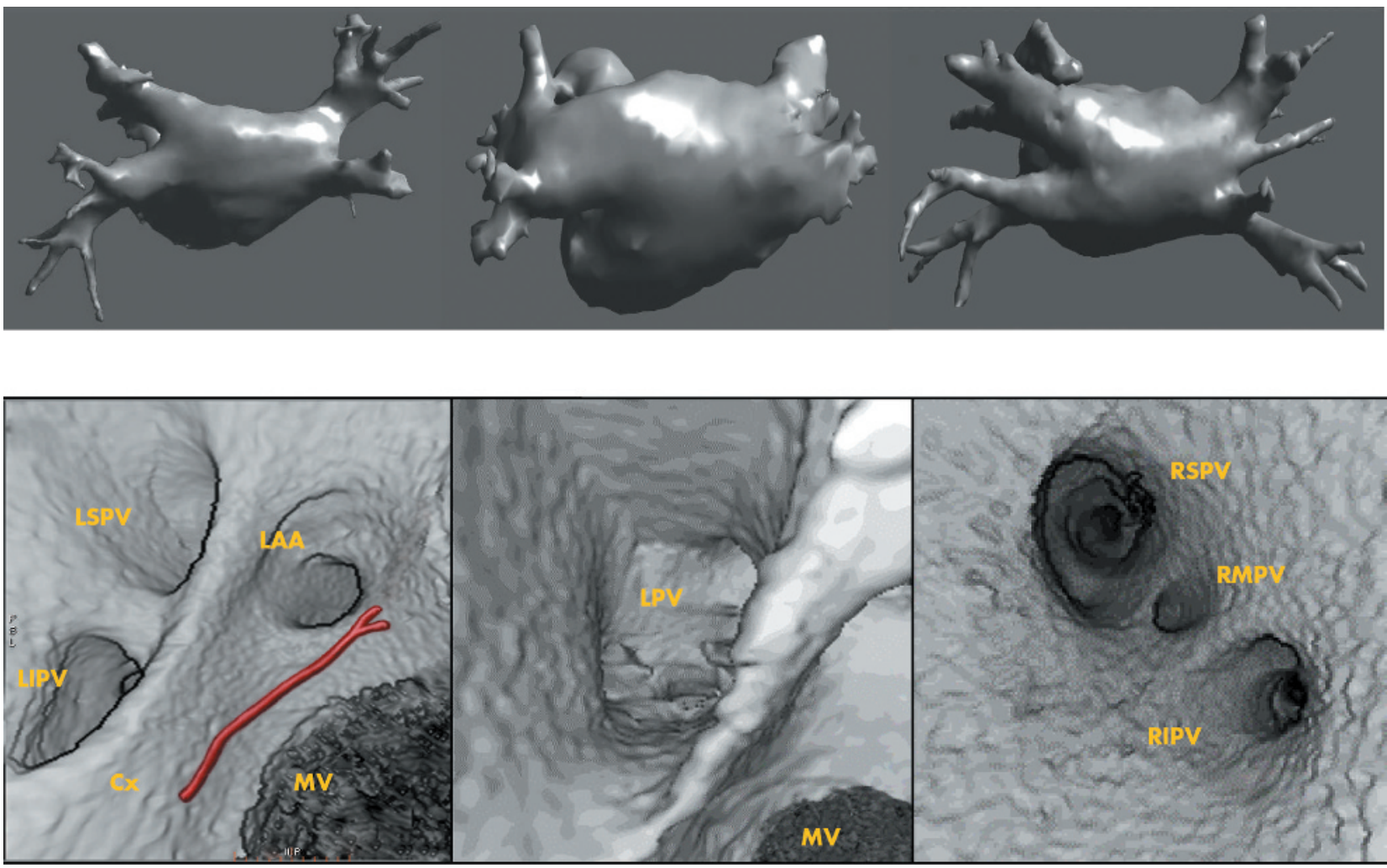

Figure 1 Three dimensional (3D) and endocardial image reconstruction: representative examples of 3D models of three different pulmonary vein morphologies along with endocardial views are depicted. As opposed to the standard four veins in the left panel, the middle panel shows a common left pulmonary vein and the right panel shows an additional right middle pulmonary vein. The endocardial views of the left and right pulmonary veins in the respective panels along with the mitral valve $(\mathrm{MV})$ are shown in the bottom panel. The location of the circumflex artery $(\mathrm{Cx})$ is also depicted in the left endocardial view. LAA, left atrial appendage; LIPV, left inferior pulmonary vein; LPV, left pulmonary vein; LSPV, left superior pulmonary vein; RIPV, right inferior pulmonary vein: RMPV, right middle pulmonary vein; RSPV, right superior pulmonary vein. Reproduced from Sra et al, ${ }^{16}$ with permission of the publisher.

The newer CT scanners are capable of imaging an organ such as the heart. ECG gated reconstruction, followed by segmentation of a 3D anatomical model, allows imaging of the heart from a particular phase of the cardiac cycle, thus effectively freezing the cardiac motion. In our institution, before AF ablation procedures, patients are scanned with contrast enhanced CT (lightspeed ultra, GE Healthcare, Waukesha, Wisconsin, USA). Non-ionic contrast is injected at $4 \mathrm{ml}$ per second, acquisition protocol: $120 \mathrm{kV}, 300 \mathrm{ma}$, 0.385 helical pitch, 0.55 second/gantry rotation, slice thickness $0.625-1.25 \mathrm{~mm}$. All images are acquired during a single breath hold. Retrospective reconstruction of the axial image set is then performed and the image dataset transferred over a network connection to a workstation (Advantage Windows, GE Healthcare) in our electrophysiology laboratory. The axial images are analysed to find data from the cardiac cycle which yields the best image quality. Typically we perform the reconstruction of the LA model at approximately $75 \%$ of the cardiac cycle (atrial diastole). ${ }^{12}$ Due to short and variable R-R intervals, a phase location at about $45 \%$ of the cardiac cycle yields the best image quality for patients in AF at the time of image acquisition. From the reconstructed data, a 3D image, or volume, of the LA chamber can be segmented successfully by processing the axial image dataset on the Advantage Windows workstation with software (CardEP, GE Healthcare) that is capable of segmentation reconstruction of 3D LA images.

LA volumes are based on the boundary between the blood pool, which is high in contrast, and the endocardium which is not contrast enhanced. This allows for clear differentiation between the atrial lumen and the atrial wall. Detection of the boundary between the blood pool and the endocardium is performed automatically using thresholding algorithms. The image is then refined manually by removing cardiac structures not included in the LA model. The LA model can be seen as a $3 \mathrm{D}$ volume, endocardial view, or navigator view. These images, in addition to providing a road map for ablation, can also be used for registration as described later in this review. Multiple variations of PV anatomy were seen in 44 consecutive patients undergoing $\mathrm{CT}$ scanning before AF ablation The variations included different sizes of PVs, a common right or left PV ostium, and an additional PV. A representative example of three different variations are depicted in fig $1 .{ }^{16}$

\section{REGISTRATION METHODOLOGY}

The registration process determines the geometric transformation that aligns fiducial points or anatomical features, also called fiducial features, in one view of an object with the corresponding points or anatomical features in another view of that object. The view can include a 3D model. The use of two different modalities on the same subject is referred to as intrasubject multimodality registration.

The number of parameters needed to describe a registration-transformation is referred to as the number of "degrees of freedom". During registration, the assumption is made that the anatomy of the organ being registered has not 
changed. The organ will thus behave as a rigid body. In this case, three translations and three rotations, which give six degrees of freedom, will lead to a successful registration. As voxel size in the system being registered may not be known, each modality, such as the CT and the interventional system, will need to be calibrated with respect to the other. This will require three extra degrees of freedom in the $3 \mathrm{D}$ space, equating to the scaling in each direction.

Fiducial points can be marked on the CT LA model as well as on the interventional system being used for registration (fig 2). Although many fiducial points can be used, at least three noncollinear points are preferred. The 3D image with marked fiducial points can be transferred in a digital format such as a DICOM file to the interventional system being used for registration. If a set of corresponding points can be identified, the registration can be affected by executing a transformation that will align these fiducial points between the two systems. Geometric transformation is a mathematical form of geometric mapping which can be used to align fiducials in two spaces or views. Each view can be referred to as a coordinate system, which will define a space for that view. If $\mathrm{x}$ and $\mathrm{y}$ are the selected two corresponding fiducial points in the two systems, the registration error $(\mathrm{T})$, also known as the fiducial registration error, will be any non-zero displacement of $\mathrm{T}(\mathrm{x})-\mathrm{y}$. Fiducial registration error, a measure of any fiducial misalignment, is a root mean square error. Optimum registration is achieved by minimising fiducial registration error. To minimise the difference between the two coordinate systems, one coordinate system is rotated and translated with respect to the other. This minimisation problem is well known and is called the orthogonal Procrustes problem. ${ }^{17}$ Procrustes was a robber in Greek mythology who altered his guests by stretching or hacking off their legs to fit the bed to which they were tied until the hero Theseus put a stop to his practices. The problem of stretching and fitting between two coordinate systems has become much more important in the field of transformation. A transformation matrix is used to map points from one coordinate space into another. By identifying the contents of the transformation matrix, several standard operations such as translation, rotation, and scaling, can be performed, essentially stretching one coordinate system into another.

A translation factor can be achieved by using techniques and processes to align the centre of the anatomical structures. Proper rotation can be calculated in terms of three angles $(\theta x$, $\theta y, \theta z)$. Assuming that the systems used for registration are calibrated such that the patient centred referential is the same in terms of orientation, there will be minimal rotation between the two systems. However, some rotational error may still occur due to movement of the heart. This can be corrected by performing registration with the interventional system at the same point in the cardiac and respiratory cycles as was used for CT scanning.

As opposed to translation and rotation, scaling can be more complex. As an example, the scaling factor $\lambda$ can be determined through known information and discernible features of a catheter. The apparent catheter electrode diameter from an exported image can be compared with the known actual dimensions of the catheter. From this information, the actual dimensions of a $3 \mathrm{D}$ model in the exported image may be determined and compared with the image structure in the interventional system.

The registration process can be executed once the previously mentioned parameters are identified. The degree of interaction between the two images is usually

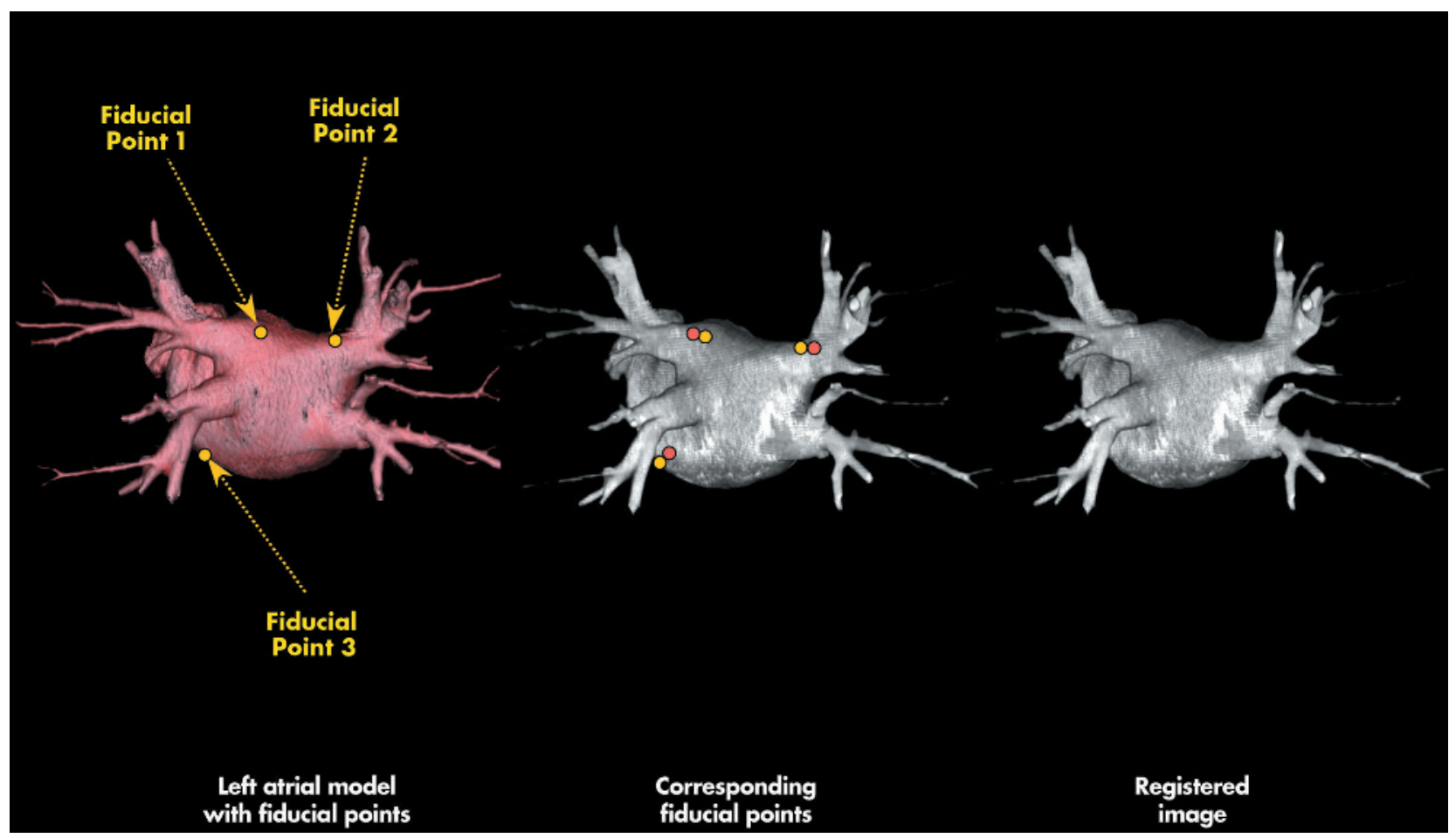

Figure 2 Registration using fiducial points. A sequence of 3D left atrial images depicting initially deposited fiducial points (yellow) during computed tomography imaging and segmentation is shown in the left panel. Following exportation of the 3D left atrial model with the fiducial points to an interventional system, a second set of corresponding fiducial points (red circles) deposited using a mapping catheter is shown in the middle panel. The coordinate system is rotated and translated to align the fiducial points and the registration process is executed, resulting in the registered image shown in the right panel. 
semiautomatic, where a computer determines the transformation and operator interaction is needed for selection of appropriate images.

In another approach, in place of internal fiducial points, external fiducial markers, anatomical features or fiducial features plus an instrument such as a catheter placed in an anatomical feature can be used for registration. Other measures, such as intensity based methods, can be used as an alternative to fiducial points or fiducial features as a registration basis. Registration, in this case, will involve calculating a transformation using voxel intensity values. Voxel similarity measures can then be calculated for the set of voxels in the overlapping region. If there is no misalignment between the images, the square of the intensity difference will be zero. This method is useful in assessing any change in pathology seen while registering serial magnetic resonance image scans. ${ }^{18}$ In the case of registration using cardiac images, a simple cost function, assuming a linear relation between the voxel intensities, may thus not be useful.

\section{REGISTRATION OF 3D MODELS WITH AN INTERVENTIONAL SYSTEM}

Recently we have studied the feasibility and accuracy of registering 3D LA models obtained with CT imaging with the non-contact mapping system. ${ }^{12}$ The non-contact mapping system utilises a multielectrode array catheter and a mapping and ablation catheter. The non-contact mapping system specifies the roving catheter position relative to the centre and orientation of the multielectrode array catheter in Cartesian coordinates. In a recent study, we performed ECG gated, contrast enhanced, cardiac CT imaging in 14 patients undergoing AF ablation. The 3D LA models were registered with non-contact mapping using fiducial points. In the first 10 patients, registration was performed retrospectively; the catheter navigation was visualised from the recorded data. A fiducial point on the cardiac model was then selected to identify the estimated location of the catheter. This process was repeated until at least three fiducial point pairs were defined. In the last four patients, registration was performed in real time during the electrophysiology study. The location of the catheter, as it was navigated in the registered model, correlated with fluoroscopy, angiography, and intracardiac recordings. The endocardial potentials occurring during sinus rhythm and any premature atrial contractions were also successfully delineated.

In a subsequent validation study using animal models, we implanted temporary pacing leads in the LA of 10 dogs. As during the feasibility study, CT scanning, segmentation, and LA model registration was performed using multiple fiducial points. Following registration, the catheter was navigated to each buried electrode, according to the registered model, with no fluoroscopic guidance. A radiofrequency lesion was delivered and the dogs sacrificed. Following staining of the heart, the distance between the buried lead and the lesion (target registration error) was 2.0 (3.6) $\mathrm{mm}$. Based on these results, it was concluded that registration of the LA, using two modalities, is feasible and accurate enough to be useful clinically.

Recently, image integration between 3D CT images and electroanatomic mapping data has been attempted. ${ }^{19}$ An electroanatomic map of the LA was acquired and displayed in the same orientation as the CT model. The CT and the electronically mapped images were then merged by matching corresponding anatomic reference points. The benefit of this approach is that it provides the operator with much more detailed simultaneous anatomic information than is available with conventional electroanatomic mapping. Although this is more advanced than standard electroanatomic mapping, it is not a true registration, as the coordinate systems from the two modalities are not synchronised. Furthermore, a detailed electroanatomic map is needed before integration of the two images can take place.

Another approach, introduced by Packer et al, ${ }^{20}$ includes integration of data from electroanatomic or non-contact mapping, CT anatomy, and intracardiac ultrasound. In a recent study on five patients, CT images of the LA were created. Ultrasound images of the PVs were generated with an intracardiac, phased array, ultrasound catheter with imaging at 5.5-10.0 MHz. A fused CT ultrasound activation sequence was created from image set. On this framework, electroanatomic or non-contact mapping was demonstrated. The images obtained with this technique are quite impressive. However, large scale use of this technique is not yet available. Use of multiple modalities may make this approach complex and the integration system expensive.

In another study by Reddy et al, ${ }^{21}$ both in vitro and in vivo experiments demonstrated the feasibility of integrating 3D magnetic resonance imaging with electroanatomic mapping data to guide real time left ventricular catheter manipulation. In this study series, initially, multiple points were obtained from a plastic model of the aorta and the left ventricle using the electroanatomic mapping system to integrate the images. This phase of the study demonstrated that registration, using points from the left ventricle only, yielded unacceptable rotational errors. These errors were satisfactorily corrected when additional points in the aorta were used for image integration. To determine the in vivo accuracy of the registration process, the image integration strategy was also assessed in a porcine infarct model. In this phase of the study, iron oxide, which is also visible on the magnetic resonance images, was injected into several sites in the ventricle before imaging. After integrating these images with the electroanatomic mapping system, ablation lesions were directed at the sites of iron oxide injections. The registration error in the study was acceptable. It is unclear how these results will pan out clinically as multiple points in the aorta and the left ventricle were needed to integrate the images. As pointed out by the authors, this, along with manual segmentation, may be time consuming. Extensive manipulation of the catheter within the aorta could be difficult in patients with significant atherosclerotic disease. Because of significant movement of the left ventricle, it is unclear how synchronisation of the image was achieved between the two systems. Despite these limitations, the results of this study were encouraging as ablation lesions were in close proximity to the infarct zone when aortic points were taken into consideration. Further study in a clinical setting is needed to validate these data.

\section{REGISTRATION OF CT LEFT ATRIAL MODELS WITH PROJECTION IMAGES OF FLUOROSCOPY SYSTEM}

Fluoroscopy is the most common interventional system used to navigate catheters and leads to appropriate sites. However, anatomical structures such as the LA and PVs are not normally delineated by fluoroscopy because there is no inherent contrast differentiation between them and the surrounding anatomy. By using a 3D imaging modality and registering these images with the projection images from the 


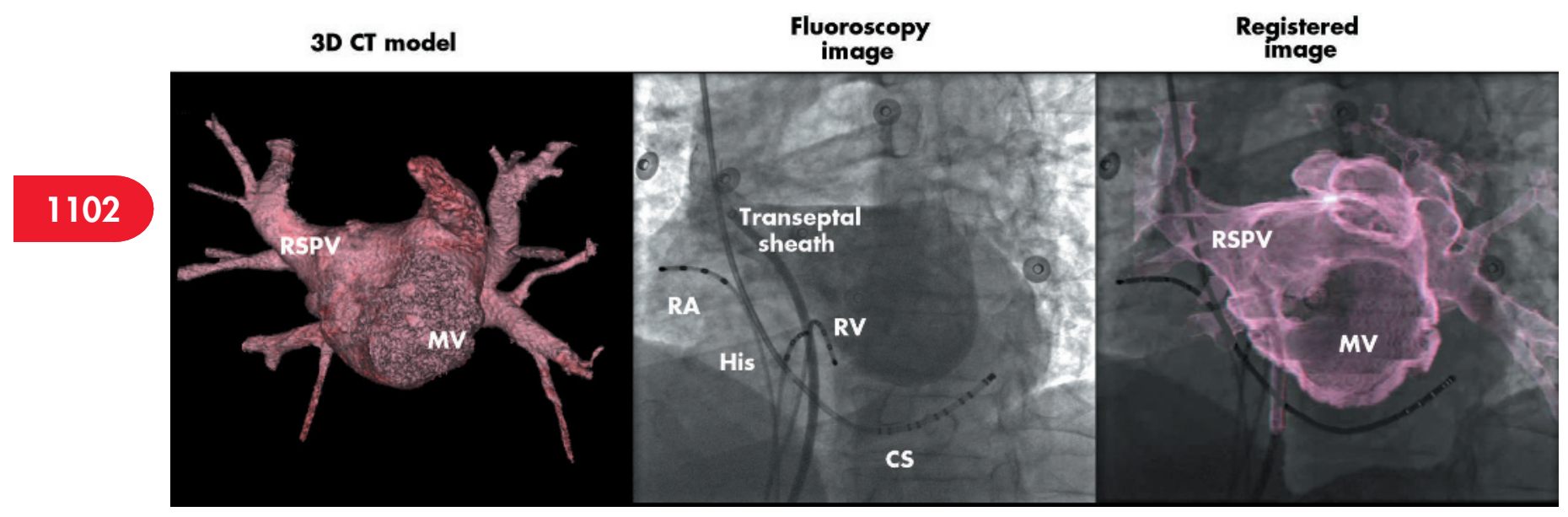

Figure 3 Registration of 3D left atrial model with fluoroscopy image. Left atrial 3D model in panel A is created using contrast enhanced ECG gated computed tomography (CT) image. During the electrophysiology procedure, digital cine sequence of the heart and surrounding structures with various catheters are obtained (panel B). Contrast is injected into the left atrium to show the outline of the left atrium, but is not needed for registration. Successful registration of the 3D left atrial image from the CT with projection image from fluoroscopy is shown in panel C. All images are shown as left anterior oblique views. See fig 1 caption for explanation of abbreviations.

fluoroscopy system, PVs and other areas in the atrium can be distinguished from the surrounding cardiac anatomy.

The image formation model of a fluoroscopy system is a conic projection. The location of the centre of the projection and the position of the imaging plane are, in principle, well described with respect to an anatomical reference system such as the patient table, for example. This anatomical reference system correlates with that used to position the patient to obtain the 3D model from the CT system. Assuming that an established protocol is followed by the operating technician during the positioning of the patient in the two acquisition systems, the CT scanner and the fluoroscopy system, the anatomical reference system may then be considered common to both acquisition systems. Especially in terms of orientation and rotation, differences between the two systems may be negligible.

A coronary sinus catheter positioned through the superior vena cava is routinely placed during AF ablation procedures. This catheter can be used both for registration and for calculation of scaling for CT fluoroscopy registration.

During pre-procedure CT imaging and segmentation, the intensity of the voxels can be adjusted to reconstruct the superior vena cava and the coronary sinus along with the LA model. Once these adjustments are made, registration with the fluoroscopy can be performed in the exported 3D model by using a transformation to align the coronary sinus catheter, seen on the fluoroscopy, with the superior vena cava and the coronary sinus in the exported 3D model. In addition to using the known and apparent diameter of the catheter identified over the registered image, scaling can be determined through the use of the scaling factor, a feature unique to the fluoroscopy system. The coronary sinus catheter is placed at a location identified by the intersection of a horizontal plane and the line joining the focal point of the fluoroscopy image. The horizontal plane is defined by an elevation above the anatomical reference system-for example, the patient table. Once the location of the catheter and the actual and apparent dimensions of the elevation in the 3D model are known, the actual size of the same anatomical structure in the 3D and the fluoroscopy image may be determined. By comparing the actual and apparent size of the anatomical structure in the LA 3D model, a scaling factor can be established between the first and the second image acquisition system, in this instance the CT model and the fluoroscopy system.

In a recent feasibility study, we performed registration of 3D LA images obtained with CT with projection images of the same obtained from fluoroscopy. In this study, 20 consecutive patients underwent contrast enhanced, ECG gated CT scanning. LA volumes were segmented from reconstructed data, as described before. During the electrophysiology procedure, digital cine sequences were obtained on the fluoroscopy system.

Rather than aligning the fiducial points, registration of the CT model and the projection image of the fluoroscopy system was performed by superimposing the coronary sinus catheter seen on the digital cine image over the superior vena cava and the coronary sinus from the CT model (fig 3). The coronary sinus catheter was also used to determine the motion of the heart and register the LA 3D model in the same phase of the cardiac cycle as segmentation reconstruction of the CT image. Successful registration was confirmed using: (1) recordings from a multielectrode basket catheter, and (2) injection of contrast into the PVs to compare overlapping of contrastfilled PVs with corresponding vessels on the registered images (fig 4). These findings may have significant clinical implications as fluoroscopy is the most commonly used interventional system, available in all electrophysiology laboratories. This form of registration could thus be potentially accessible to most laboratories. It would enable more appropriate navigation and localisation of the mapping and ablation catheters.

\section{CONCLUSIONS}

Image registration involves correspondence between two images or an image and a physical space. Proper imaging and segmentation involves establishing a correspondence between the spatial information in the image and the equivalent anatomical structure in the body. Careful application of these data is necessary to avoid any misregistration leading to error in the delivery of treatment. Improvements in imaging and computer technology have led to successful 


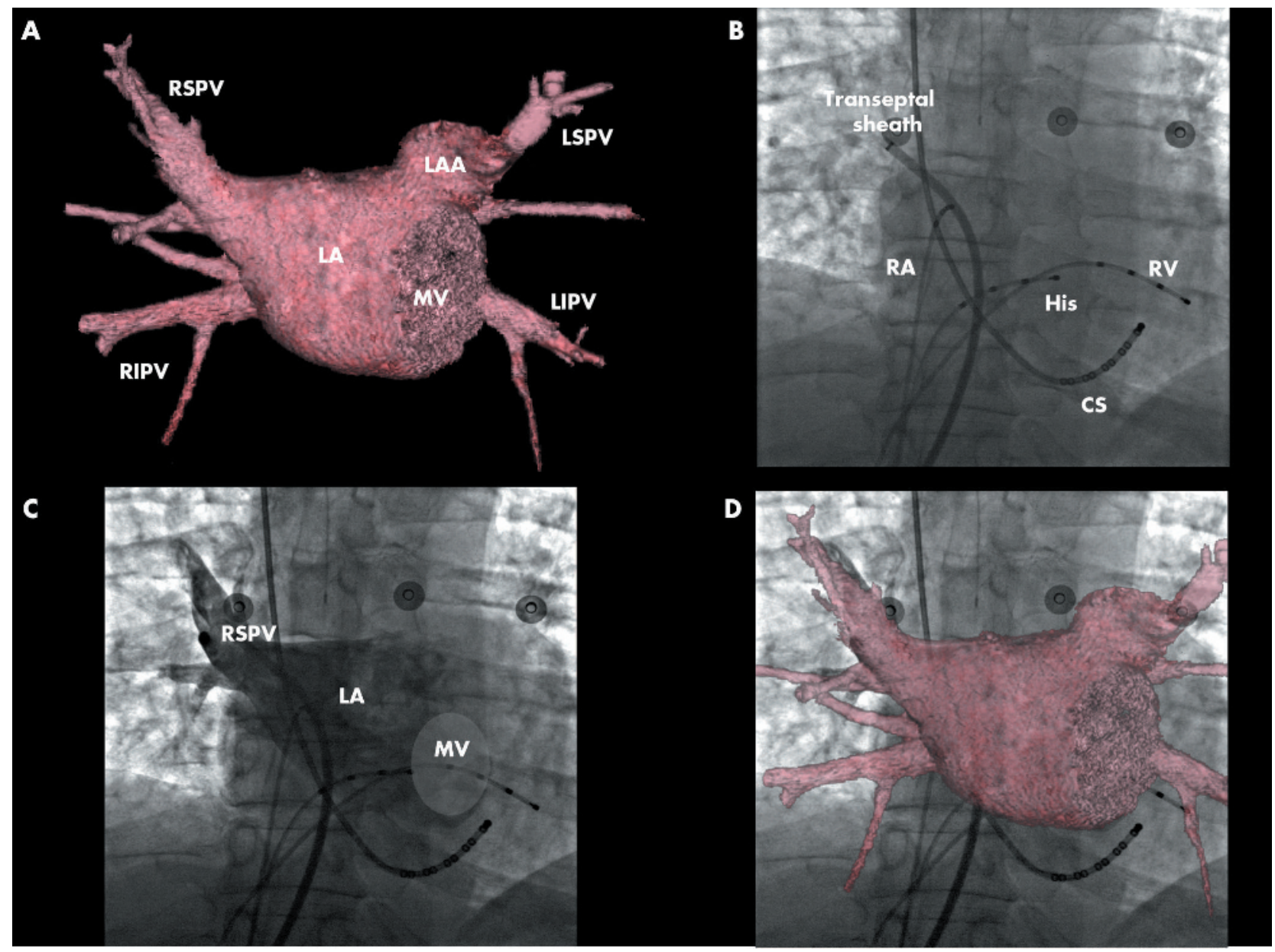

Figure 4 Validation of registered images. Following registration of 3D left atrial computed tomography model in panel A with the projection images of fluoroscopy in panel $B$, contrast is injected into the right superior pulmonary vein to validate the registration. Panel $C$ is a translucent view of the registered image showing the contrast filled right superior pulmonary vein more appropriately. There is overlapping of the registered and contrast filled vein suggesting appropriate registration. Panel D shows registered 3D volume of the CT with the projection images of fluoroscopy. See fig 1 caption for explanation of abbreviations.

registration of cardiac models through the use of two or more different modalities. Clinical availability of such a system could, potentially, be of great utility and have broad applicability in the treatment of cardiac arrhythmias. Steps involved in the registration process can be made simpler by making them automatic.

Additional references appear on the Heart website-http:// www.heartjnl.com/supplementa

Competing interests: JS has been reimbursed by Endocardial Solutions, Inc, Biosense Webster, Inc, and GE Healthcare for attending several conferences; JS has been paid by Endocardial Solutions, Inc, Biosense Webster, Inc, and GE Healthcare for running educational programmes and has received funds from them for research. In compliance with EBAC/EACCME guidelines, all authors participating in Education in Heart have disclosed potential conflicts of interest that might cause a bias in the article

\section{REFERENCES}

1 Hajnal JV, Hill DLG, Hawkes DJ. Medical image registration. The Biomedical Medical Engineering Series 2001:1-70.

- Detailed review of registration techniques and different algorithms which can be used for registration.
2 van den Elsen PA, Pol EJD, Viergever MA. Medical image matching: a review with classification. IEEE Eng Med Biol 1993:2:26-39.

3 Haissaguerre $M$, Jais $P$, Shah DC, et al. Spontaneous initiation of atria fibrillation by ectopic beats originating in the pulmonary veins. N Engl J Med 1998;339:659-66.

- The author found that ectopic beats, originating from the superior pulmonary veins predominantly, initiated paroxysms of atrial fibrillation, and that these veins could be electrically isolated by targeting the sites of double potentials, thus suggesting the electrical connection between the left atrium and the pulmonary veins.

4 Chen SA, Hsieh MH, Tai CT, et al. Initiation of atrial fibrillation by ectopic beats originating from the pulmonary veins: electrophysiological characteristics, pharmacological responses, and effects of radiofrequency ablation. Circulation 1999:100:1879-86.

5 Pappone C, Rosanio S. Oreto G, et al. Circumferential radiofrequency ablation of pulmonary vein ostia: a new anatomic approach for curing atrial fibrillation. Circulation 2000;102:2619-28.

- This study was designed to assess the efficacy and safety of circumferential pulmonary vein isolation using an electroanatomic mapping system. Unlike the study by Haissaguerre et al, no attempts were made to isolate the pulmonary veins electrically.

6 Ben-Haim SA, Osadchy D, Schuster I, et al. Nonfluoroscopic, in vivo navigation and mapping technology. Nat Med 1996;2:1393-5.

7 Wittkampf FH, Wever EF, Derksen R, et al. Localisa: new technique for realtime 3 dimensional localization of regular intracardiac electrodes. Circulation $1999 \cdot 99 \cdot 1312-7$

8 Peters NS, Jackman WM, Schilling RJ, et al. Human left ventricular endocardial activation mapping using a novel noncontact catheter. Circulation 1997:95:1658-60.

9 Sra J, Hauck J, Krum D, et al. Three-dimensional right atrial geometry construction and catheter tracking using cutaneous patches. J Cardiovasc Electrophysiol 2003; 14:897. 
10 Lacomis JM, Wigginton W, Fuhrman C, et al. Multi-detector row CT of the LA and pulmonary veins before radio-frequency catheter ablation for atrial fibrillation. Radiographics 2003;23:S35-50.

- The dimensions of the left atrium and pulmonary veins were determined using the multidetector helical computed tomography system.

11 Kato R, Lickfett L, Meininger G, et al. Pulmonary vein anatomy in patients undergoing catheter ablation of atria fibrillation: lessons learned by use of magnetic resonance imaging. Circulation 2003;107:2004-10.

- The study documented that patients with AF have larger pulmonary veins than controls and demonstrated the value of magnetic resonance imaging in AF ablation.

12 Sra J, Krum D, Hare J, et al. Feasibility and validation of registration of three-dimensional left atrial models derived from computed tomography with a noncontact cardiac mapping system. HeartRhythm 2005;2:55-63.

- The study demonstrated successful registration of a CT left atrial model with a non-contact mapping system in patients and animal models.

13 Webb S. The physics of medical imaging. Bristol: Institute of Physics Publishing, 1988

14 Peters TM, Clark JA, Olivier A, et al. Integrated stereotaxic imaging with CT, MR imaging, and digital subtraction angiography. Radiology 1986;161:821-6.

15 Roberts DW, Strohbehn JW, Hatch JF, et al. A frameless stereotaxic integration of computerized tomographic imaging and the operating microscope. J Neurosurg 1986;65:545-9.

16 Sra J, Krum D, Okerlund D, et al. Endocardial imaging of the left atrium in patients with atrial fibrillation. J Cardiovasc Electrophysiol Images 2004; 15:247.

17 Schönemann PH. A generalized solution of the orthogonal Procrustes problem. Psychometrica 1966;31:1-10.

18 Hainal JV, Saeed N, Soar EJ, et al. A registration and interpolation procedure for subvoxel matching of serially acquired MR images. J Comput Assist Tomogr 1995; 19:289-96.

- The authors demonstrate the efficacy and usefulness of serial registration techniques using a single modality to assess and compare any changes in pathology.

19 Verma A, Marrouche N, Natale A. Novel method to integrate threedimensional computed tomographic images of the left atrium with real-time electroanatomic mapping. J Cardiovasc Electrophysiol 2004;15:968.

- First case report demonstrating integration of a detailed left atrial image created using an electroanatomic mapping system with the CT left atrial model.

20 Packer DL, Monahon KH, Peterson LA, et al. Integration of multimodality 5D mapping for atrial arrhythmias. Pacing Clin Electrophysiol 2003;26:107.

- Left atrial data from electroanatomic or non-contact mapping was successfully integrated with $\mathrm{CT}$ anatomy and ultrasound.

21 Reddy VY, Malchano ZJ, Holmvang G, et al. Integration of cardiac magnetic resonance imaging with three dimensional electroanatomic mapping to guide left ventricular catheter manipulation: feasibility in a porcine model of healed myocardial infarction. J Am Coll Cardiol 2004;44:2202-13.

- In vitro and in vivo experiments demonstrating the feasibility of integrating 3D magnetic resonance imaging with electroanatomic mapping data to guide real time left ventricular catheter manipulation.

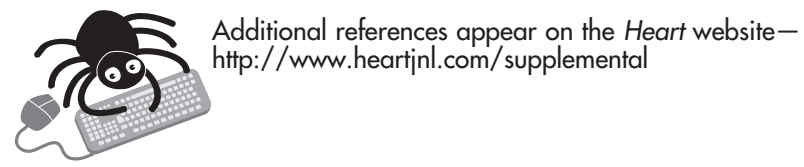

\section{MULTIPLE CHOICE QUESTIONS}

Education in Heart Interactive (www.heartinl.com/misc/education.shtml)

There are six multiple choice questions associated with each Education in Heart article (these questions have been written by the authors of the articles). Each article is submitted to EBAC (European Board for Accreditation in Cardiology; www.ebac-cme.org) for 1 hour of external CPD credit.

How to find the MCQs: Click on the Online Learning: [Take interactive course] link on the table of contents for the issue online or on the Education in Heart collection (www.heartjinl.com/cgi/collection/heart_education).

Free access: This link will take you to the BMJ Publishing Group's online learning website. Your Heart Online user name and password will be recognised by this website. As a Heart subscriber you have free access to these MCQs but you must register on the site so you can track your learning activity and receive credit for completed courses.

How to get access: If you have not yet activated your Heart Online access, please do so by visiting http://www.bmijournals.com/cgi/activate/basic and entering your six digit (all numeric) customer number (found above your address label with your print copy). If you have any trouble activating or using the site please contact subscriptions@bmigroup.com

Case based Heart: You might also be interested in the interactive cases published in association with Heart (http://cpd.bmijournals.com/cgi/hierarchy/cpd_node;CBH) 